\title{
Engaging Activities for Enhancing Mathematical Learning
}

\author{
Natali Hritonenko ${ }^{1,}{ }^{*}$, Victoria Hritonenko ${ }^{2}$ and Olga Yatsenko ${ }^{3}$
}

\author{
${ }^{1}$ Prairie View A\&M University, Texas, USA \\ ${ }^{2}$ UC Berkeley Extension, California, USA \\ ${ }^{3}$ UT Health, Texas, USA \\ *Corresponding author. Email: nahritonenko@pbvamu.edu
}

\begin{abstract}
Challenges in mathematical education have been recognized throughout the world. Mathematical courses are among the most difficult subjects and a barrier for students in pursuit of their career dreams. Captivating puzzles and cross disciplinary projects are presented in this paper. They contribute to novel pedagogical strategies to foster students' understanding of mathematical foundations and relations among different mathematical topics Such extracurricular activities reduce mathematical anxiety, spark interest in the subject, and enrich students with mathematical fundamentals. They can be incorporated to a great variety of mathematical courses and are proven to work well in face-to-face and virtual classroom settings.
\end{abstract}

Keywords: Mathematics education, Extra-curricular activities, Puzzle, Projects.

\section{INTRODUCTION}

College Algebra is a core or prerequisite course in most college degrees. Finite Mathematics, Business Mathematics, Mathematics for Biology, Contemporary Mathematics, and other subject-oriented algebrasatellite courses are offered by different colleges to better serve their majors and increase students' success. Tutorial services, excellent easy-to-read textbooks accompanied with practical software, and a plethora of YouTube videos, animations, and tutorials are available for students to assist them in their studies. However, despite these improvements in accessibility to resources, no significant improvement has been made. A recent report by the Mathematical Association of America [1] stated that over $50 \%$ of students nationwide fail College Algebra.

Several extra-curricular activities are suggested and discussed in this paper. They are proven to work well in both face-to-face and virtual classroom settings. Projects and puzzles are widely used education techniques [2-7], but their presented variations go beyond traditional tasks and surpass routine homework. A fascination collection of 325 cross-disciplinary problems presented in [8] is also a valuable asset for mathematical courses. Captivating and exciting decode-a-phrase sudoku and interdisciplinary assignments are designed to reduce mathematical anxiety, stimulate learning of mathematical fundamentals, and demonstrate the versatility of mathematics and unity of science. Educational elements are integrated into all these activities that can be easily incorporated into any mathematics or similar courses across disciplines. All presented activities are accompanied with detailed descriptions. These activities do not require any mathematical proficiency above College Algebra, but, rather, deeper thinking and understanding. Indeed, most of these activities can be completed solely relying upon basic mathematical knowledge from middle and high school!

The next section discusses games and puzzles that can be incorporated to any class and presents a statistical Sudoku as an example. The third section offers examples of interdisciplinary projects and emphasizes their importance. The last section concludes.

\section{GAMES AND PUZZLES}

People of all ages enjoy playing games and solving puzzles. Modified and integrated into a course, games and puzzles become a powerful educational tool that has advantages over traditional instruction, significantly stimulates and inspires students to study, and encourages a positive learning attitude. Students learn 
by playing. Targeted basic mathematical rules are reviewed, repeated and, finally, memorized in topicoriented games [3,4,5]. Math Bingo, Guess What, Sudoku, and other educational games greatly contribute to successful studying. Such activities provide numerous benefits for both instructors and students [9]. Instructors can identify and retain the most advanced and active students in a group, discover students' potentials, and enhance deeper understanding of a subject.

All extra-curricular activities should be flexible, especially in online classes. Depending on the group of students, their ages and interests, different games and puzzles can be designed and incorporated into course curriculum. If participation of students in one game is low, another one can be offered. What works well for one group may not work for another. A small questionnaire at the beginning and during a course can direct an instructor to choose a right game or puzzle for a certain group. According to students' surveys, most of them like sudoku, kakuro, suguru, inkies, and other number puzzles and enjoy exploring new ones. Plenty of different puzzles, e.g., [8], can be modified to desirable topics and outcomes. In this section, we concentrate on sudoku, though alternative games and puzzles can be used if students prefer them. Every sudoku has a unique solution and can be solved through logic alone. Nine symbols, usually numbers from 1 to 9 , should be entered into the blank spaces so that each row, column, and 3x3 box contain the symbols without repetition.

Below we provide an example of special sudoku, in which letters are used as symbols instead of commonly used numbers. Students must solve the puzzle and then read a decoded phrase using the provided hints.

\subsection{Statistical Sudoku}

Target Topic: Introduction to Statistics and Probability

Task: Enter a letter from the set $\{\mathbf{A}, \mathbf{C}, \mathbf{I}, \mathbf{S}, \mathbf{T}, \mathbf{V}\}$ into the blank cells in Table 1 so that each letter appears only once in each row, column, and 2x3 small square.

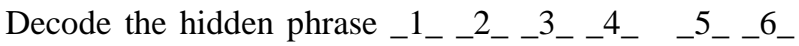
_7__8__9__10__11__12__13__14_,

where each number stands for a letter from the sudoku that satisfies the statements below.

Table 1 Statistics Sudoku

\begin{tabular}{|c|c|c|c|c|c|}
\hline S & & & & & C \\
\hline & T & & & & I \\
\hline & & A & & S & \\
\hline & S & & T & & \\
\hline I & & & & A & \\
\hline V & & & & & S \\
\hline
\end{tabular}

1. Symbol 1 is in the cell where the row is the standard deviation of the dataset $\{2,4,4,4,5,5,7,9\}$ and the column is its mean.

2. A dataset with the mean of 3 and standard deviation of 4 is considered. Then, 3 is added to each observation. Symbol 2 is at the intersection of the row and column presented by the mean and standard deviation of the new dataset, respectively.

3. Symbol 3 is located at the intersection of the row represented by ten times probability and the column by ten times probability $P(\overline{A \cup B})$ and the column represented by ten times $P(\bar{A} \cup \bar{B})$ if $P(A)=0.6$, $P(B)=0.7, P(A \cap B)=0.5$, given that events A and $\mathrm{B}$ are from the same sample space.

4. The row and column of Symbol 4 are the same. If the statement "The variance of a dataset is greater than its standard deviation" is correct, their values are 1 ; if it is incorrect, their values are 2; and if there is not enough information to answer, their values are 3.

5. A dataset with the mean of 2 and median of 4 is considered. Two observations are added to the dataset. The first added observation is 2 less than the smallest observation in the dataset and the second one is 2 more than its largest observation. Symbol 5 is located at the row represented by the mean and column represented by the median of the new data set.

6. Symbol 6 is at the intersection of the row that is the total sum of all observations and the column that is the number of all observations of a data set with the following properties:

- the total sum of all observations divided by the number of observations is 2 ;

- the total sum of all observations is equal to 3 times the mean of the dataset.

7. Symbol 7 is at the intersection of the row and column represented by the mean and standard deviation of a new dataset obtained from the dataset with the mean of 1 and standard deviation of 2 after each observation is multiplied by 3 and then increased by 1 .

8. Symbol 8 is at the intersection of the row and the column, both represented by ten times $P(A \cap B)$ if $P(A)=0.7, P(B)=0.6$, and $P(A \cup B)=0.9$.

9. The row of Symbol 9 is the numerator and its column is the denominator of an irreducible fraction that stands for the probability that two distinct letters from the set $\{\mathrm{A}, \mathrm{C}, \mathrm{I}, \mathrm{S}, \mathrm{T}, \mathrm{V}\}$ appear in one row of sudoku.

10. Symbol 10 has the row equal to ten times and the column equal to ten times if $\mathrm{A}$ and $\mathrm{B}$ are two disjoint events from the same sample space, $P(A)=0.2$, and $\mathrm{P}(\mathrm{B})=0.3$. 
11. Symbol 11 is at the intersection of the row and column represented by the mean and median of a new dataset obtained by adding 2 to each observation in a dataset with the mean of 1 and median of -1 .

12. Symbol 12 is located at the intersection of the row and column represented by the mean and mode of the dataset $\{2,3,2,4,2,5\}$, respectively.

13. Symbol 13 can be found from the problem: "There are teenagers in a room with the average age of 16 and the standard deviation of 2 . One of the teenagers aged 16 leaves the room. In what way do the mean and standard deviation change?”

Take

- " 1 ” for the row of Symbol 13 if the new mean decreases,

- "2” if the mean stays the same,

- “ 3 ” if the mean increases,

Take

- " 1 " for the column if the new standard deviation decreases,

- "2" if the new standard deviation stays the same,

“ 3 ” if the new standard deviation increases.

14. A box contains 10 green, yellow, and red balls. The probability of selecting a red ball is 0.2 , while the probability of selecting a red or yellow ball is 0.5. Symbol 14 has the row represented by the number of yellow balls and the column represented by the number of green balls in the box.

Yes, indeed, VIVA STATISTICS.

\section{MATHEMATICAL PROJECTS ACROSS DISCIPLINES}

Medieval English philosopher and scholar Roger Bacon (1214-1294) stated, all sciences require mathematics...for the things of this world cannot be made known without knowledge of mathematics. It is difficult, if not impossible, to find a discipline that does not use mathematics.

Students with inadequate mathematical preparation experience difficulties in their other classes. They often do not realize why, despite their hours of study, they are not getting the grade they think they deserve. Instructors from different disciplines complain that lack of or poor mathematical preparation is the most common cause of students' failure in their class and that they have to work in both directions, covering their subject and teaching mathematical foundations needed for their class.

Cross-disciplinary strategies are among the contemporary educational approaches. Interdisciplinary projects emphasize applicability of mathematics, unity of all disciplines, and flexibility of science [9]. Such projects motivate the simultaneous learning of mathematics and other disciplines helping students discover practical reasoning behind mathematical concepts. They nurture students' knowledge and practice their skills, as students enhance themselves while studying a subject and increasing a level of their mathematical literacy at the same time. The mathematical level of cross-disciplinary projects should be adjusted depending on a subject, students' preparation level, and interests. The ideas for such projects can be taken from any paper, journal, or book.

Examples of just two cross-disciplinary projects with their motivation and tasks are shown in the next subsections. These and similar projects received high approval rating from students who mentioned numerous benefits of the projects in their evaluations.

\subsection{Environmental Sciences}

Motivation. We live, produce, and contaminate. Adaptation to climate change and mitigation are two long-term strategies for dealing with climate change, reducing pollution, and protecting our environment. Following $[10,11]$, adaptation to climate change is an adjustment in natural or human systems in response to actual or expected climatic stimuli or their effects and mitigation is an anthropogenic intervention to reduce the sources or enhance the sinks of greenhouse gases. Implementation of both policies are extremely expensive and raise numerous issues, such as finding the optimal mix of and investment to adaptation and mitigation strategies, the long- and short-term effectiveness of mitigation and adaptation, and others. Mathematical modeling provides a powerful analytic framework to address such issues. Suggested environmental models should reflect realistic properties of mitigation and adaptation and be simple enough to be used as blocks in larger models for development of environmental protection policies at national and international levels.

In modeling adaptation efficiency functions [10,11], the following properties are considered:

(i) no damage is reduced without adaptation,

(ii) infinite adaptation can reduce almost all or all damage,

(iii) the more adaptation is used, the less effective it will be.

Tasks. Consider two formulas for the adaptation efficiency function:

$\eta(D)=\frac{1}{1+D}$ 


$$
\eta(D)=\underline{\eta}+(\bar{\eta}-\underline{\eta}) e^{-a D}, \bar{\eta}>\underline{\eta}>0, a>0
$$

where $\mathrm{D}$ is the investment into adaptation, a reflects the marginal efficiency of adaptation, and are maximum and minimum values of the adaptation efficiency.

a. Provide interpretation and real examples of each property of (i)-(iii).

b. Check whether functions (1) and (2) possess properties (i)-(iii) of adaptation effectiveness.

c. Provide applied interpretation of maximum and minimum values of the adaptation efficiency.

d. Sketch the functions (1) and (2) and compare their graphs.

e. Which function (1) or (2) is more general and reflects more realistic situations?

f. Find the range of parameters when the functions (1) and (2) have similar qualitative behaviour.

g. Suggest other functions that satisfy properties (i)-(iii) and, thus, can be considered as adaptation efficiency functions.

h. Provide applied interpretation of all outcomes.

\subsection{Biological Sciences}

Motivation. Tumors are cell populations growing in a confined space where the availability of nutrients is limited. A.K. Laird suggested in the 1960s the function (3) to fit data of the tumor growth [12]:

$$
x(t)=K e^{e^{-\alpha t} \ln \frac{x(0)}{K}}
$$

In (3) $x(t)$ is the tumor size, $x(0)$ is the initial tumor size at $t=0, K$ is the carrying capacity, $a$ is a constant related to the proliferative ability of the cells.

Tasks are below:

a. Describe the function $x(t)$ and its dependence on parameters $x(0), K$ and $a$. Provide applied interpretation of your findings.

b. Sketch a graph of the function $x(t)$ described by (3). What shape does it look like? What could you call the curve described by (3)?

c. Justify that $K$ in (3) is the carrying capacity, that is, the maximum size that can be reached with available nutrients.

d. Is the function $x(t)$ one-to-one? Find its inverse if yes.

e. How much time is needed to have the initial size of cells doubled?

f. Find other applications of the function (3). g. Find other functions that have a shape similar to the function $x(t)$ described by (3).

\section{CONCLUSION}

Projects and puzzles are widely used educational practices, but their presented variations are nontraditional. A few examples of different types of activities, such as sudoku and interdisciplinary projects, that can be offered as extra credit assignments in mathematical classes and across disciplines are presented in this paper. Students are exposed to a novel approach to learning and reviewing mathematical topics. Although some activities may be challenging for some groups of students, none of them require any additional knowledge of mathematics, just deeper thinking. Such activities enhance students' abilities to recognize and apply basic algebraic rules faster, significantly improve their problem-solving skills, and boost their creativity and logical skills. Even if students do not completely succeed in a task, they will be enriched with a thoughtful understanding of the fundamental rules of mathematics and develop mathematical reasoning needed for their successful undergraduate study and beyond.

\section{AUTHORS’ CONTRIBUTIONS}

All authors worked on the paper, discussed the results, and contributed to the final manuscript. N.H. prepared designed the paper and managed the work. V.H. contributed to Sections 1 and 3. O.Y supplied and verified material for Sections 2 and 4.

\section{ACKNOWLEDGMENTS}

The authors would like to thank all organizers of DESD-2021 International Conference on Diversified Education and Social Development for their great job for making this conference to be successful.

\section{REFERENCES}

[1] Mathematical Association of America, https://www.maa.org/college-algebra, accessed November 3, 2020

[2] N. Hritonenko, Student Projects in the Educational Process DELTA-K, Journal of the Mathematics Council of the Alberta Teachers' Association, 41 (2004), No.1, 51-52.

[3] N.Hritonenko, Logical Problems in Teaching Statistics DELTA-K, Journal of the Mathematics Council of the Alberta Teachers' Association, 40 (2003), No.1, 73-76.

[4] Yu.Zhonggen, A Meta-Analysis of Use of Serious Games in Education over a Decade, International Journal of Computer Games Technology, Volume 
2019, Article ID 4797032 https://doi.org/ 10.1155/2019/4797032Ed.

[5] Campbell, N.Hritonenko, Integrated Approach in Teaching Trigonometry Concepts, Teaching Journal of the ooi Academy, 5 (2005), No 1, 121129.

[6] N.Hritonenko, Mathematics with Joy, DELTA-K, Journal of the Mathematics Council of the Alberta Teachers’ Association, 39 (2002), No.2, 9-13.

[7] N.Hritonenko, Analysis of Teaching Trigonometry in the Context of University Mathematics, DELTA-K, Journal of the Mathematics Council of the Alberta Teachers' Association, 34 (1997), No.2, 49-53.

[8] N. Hritonenko \& Yu. Yatsenko, USA Through the Lens of Mathematics, to appear in 2021, Chapman and Hall/CRC Press.
[9] Krazydad, Printable Puzzles, Mazes and More! https://krazydad.com/, accessed November 3, 2020

[10] N. Hritonenko, O. Yatsenko, Projects to facilitate mathematical learning, in Teaching mathematics in higher education and working with gifted students in contemporary context, Mogilev, Belarus, February, (2019), 8-11.

[11] Th. Bréchet, N. Hritonenko, Yu. Yatsenko, Domestic environmental policy and international cooperation for global commons. Resource and Energy Economics, 2016, 44, 183-205

[12] Th. Bréchet, N. Hritonenko, Yu. Yatsenko, Adaptation and mitigation in long-term climate policy. Environmental and Resource Economics, 2013, 55, 217-243 22.

[13] A.K. Laird, Dynamics of tumor growth. $\mathrm{Br} \mathrm{J}$ of Cancer ,1964,

18: 490-502. 